\title{
PROPRIEDADES FÍSICAS E TAXA DE ESTRATIFICAÇÃO DE CARBONO ORGÂNICO NUM LATOSSOLO VERMELHO APÓS DEZ ANOS SOB DOIS SISTEMAS DE MANEJ $0^{(1)}$
}

\author{
C. A. TORMENA(2), R. FRIEDRICH(3), J . C. PINTRO(2), \\ A. C. S. $\operatorname{COSTA}^{(2)} \&$ J . FIDALSKI ${ }^{(3)}$
}

\begin{abstract}
RESUMO
Nos solos de textura argilosa e muito argilosa, a compactação das camadas superficiais constitui uma das limitações do solo sob plantio direto. Nestas condições, tem sido adotado o revolvimento periódico do solo. As rotações de culturas são indicadas para o manejo físico do solo em razão do maior aporte de matéria orgânica e bioporosidade do solo. 0 objetivo deste trabalho foi quantificar, num L atossolo Vermel ho eutroférrico, algumas propriedades físicas, os teores e a taxa de estratificação do carbono orgânico do solo (COS), após dez anos da instalação de dois sistemas de manejo do solo. Os si stemas de manejo de solo estudados foram: plantio direto com rotação de culturas (PD) e plantio direto com sucessão de culturas, realizando a escarificação do solo antes da semeadura da cultura de verão (PDR). Maiores valores de densidade do solo foram observados no PD e de macroporosidade e porosidade total no PDR. Nos dois sistemas, o volume de macroporos foi maior que $10 \%$, indicando que as condições de aeração foram adequadas para as raízes das plantas. A curva de retenção de água indicou que o PDR retém mais água que o PD em elevados potenciais, sendo si milares para potenciais menores que -0,008 $\mathrm{MPa}$. A resistência do solo à penetração (RP) foi maior no $P D$ até à profundidade de $0,20 \mathrm{~m}$, independentemente da umidade do solo. A partir dos resultados, verificou-se que, no PD, sob condi ções si milares de secamento do solo, os valores de RP foram maiores do que no PDR, podendo atingir valores críticos ao crescimento das plantas. Assim, estratégias de manejo para conservar a umidade no solo são necessári as para manter a RP abai xo de valores impeditivos às plantas. Os teores de C-orgânico do solo foram maiores no PD até 0,10 m de profundidade, enquanto de 0,10-0,40 m, foram constatados maiores valores no PDR. A taxa média de
\end{abstract}

(1) Recebido para publicação em agosto de 2003 e aprovado em outubro de 2004.

(2) Professor Adjunto, Departamento de Agronomia, Universidade Estadual de Maringá - UEM. Av. Colombo 5790, CEP 87020-900 Maringá (PR). Bolsista do CNPq. E-mail: catormena@uem.br; jcpintro@uem.br; acscosta@uem.br

(3) Alunos do Programa de Pós-Graduação em Agronomia, UEM. E-mails: fidalski@iapar.br 


\begin{abstract}
estratificação de COS foi de 1,73 no PD, enquanto no PDR foi de 1,24 . Os resultados evidenciam que a maior concentração de COS no PD pode resultar em condições físicas mais estáveis às culturas.
\end{abstract}

\author{
Termos de indexação: plantio direto, rotação de culturas, resistência do solo à \\ penetração, densidade do solo
}

\author{
SUMMARY: SOIL PHYSICAL PROPERTIES AND ORGANIC CARBON \\ STRATIFICATION RATE IN AN OXISOL AFTER TEN YEARS \\ UNDER TWO DIFFERENT SOIL MANAGEMENT SYSTEMS
}

\begin{abstract}
In the clay and heavy clay soils, compaction of the surfacelayer represents one of the limitati ons under no-tillage. Under such conditions, periodic soil tillagehas been adopted. Other techniques, such as crop rotation are indicated for the physical management of the soil due to a larger input of organic matter and bioporosity of the soil. The objective of this study was to quantify some soil physical properties in an eutroferric Red Latosol (rhodic Eutrudox), theorganic carbon content and thesoil organic stratification rateafter ten years of two soil management systems. The soil management systems included: crop rotation under no-tillage (NT), and crop-sucession under no-tillage, but with soil chiseling before thesummer crop (CNT). Larger values of bulk density wereverified in NT and macroporosity and total porosity in CNT. In both management systems, the air-filled porosity was above $10 \%$, suggesting that the aeration conditions wereappropriatefor the plant roots. Thesoil water retention curve indicated that thesoil under CNT retains more water than under NT at high potentials, but they aresimilar at potentials bel ow $-0.008 \mathrm{MPa}$. Thesoil penetration resistance (RP) was larger in NT down to the depth of $0.20 \mathrm{~m}$, independently of the soil moisture content. Results suggested that with identical soil dryness the RP can reach restrictiveval ues for plant growth in NT. Thus, management practices to maintain thesoil moisturearenecessary to keep the RP lower than thelevel s considered restrictivefor plants. The soil organic content (SOC) was higher in NT down to a depth of $0.10 \mathrm{~m}$, while higher values were observed at depths of $0.10-0.40 \mathrm{~m}$ in CNT. The SOC stratification ratein the soil was 1.73 in NT while in CNT it was 1.24. Results show that this highest SOC concentration in NT may result in morestablesoil physical conditions for crops.
\end{abstract}

Index terms: no-tillage, crop rotation, soil penetration resistance, soil bulk density.

\section{NTRODUÇÃO}

Nos solos pertencentes às classes de textura argil osa do estado do Paraná, o avanço das culturas produtoras de grãos ocorreu com o uso de sistemas intensivos de preparo do solo. A erosão, a compactação, a perda da fertilidade e da matéria orgânica são conseqüências destes sistemas de manejo empregados einfluem na produtividade das culturas. Resultados positivos na redução da degradação dos sol os têm sido obti dos com a adoção de sistemas de plantio direto, mantendo-se os resíduos culturais na superfície do solo (Derpsch et al., 1986; Roth et al., 1988).

Com a introdução do sistema plantio dir eto, com a ausência de revolvimento e com o efeito cumulativo do tráfego de máquinas na superfície do solo, temse constatado compactação superficial do solo (Tormena \& Roloff, 1996; De Maria et al., 1999). A compactação reduz o volume total de poros do solo (Defossez \& Richard, 2002) e altera a distribuição do tamanho dos poros (Klein \& Libardi, 2002). De acordo com Boizard et al. (2002), a intensidade e a freqüência de ocorrência da compactação dependem da seqüência das culturas, do sistema de manejo e da umidade do solo por ocasião do tráfego.

A compactação acarreta conseqüências negativas na qualidade física do solo para o crescimento das plantas (Tormena et al., 1998b), em virtude das modificações del etérias na disponibilidade de água no sol o às plantas, na capacidade de difusão gasosa do solo e na resistência do solo à penetração das raízes (RP) (Boone et al., 1986). Porosidade com ar de $10 \%$ é o mínimo necessário para a difusão de $\mathrm{O}_{2}$ até às raízes (Xu et al., 1992), indicando que a redução no volume de macroporos reduz a difusão $\mathrm{de}_{2}$ no solo. Neste contexto, a disponibilidade de água no sol o entre os potenciais mátricos de -0,01 e 
-1,5 MPa, estabelecida como água disponível às plantas, tem sido utilizada no desenvol vimento ena avaliação de estratégias de manejo do solo (Cassel $\&$ Nielsen, 1986).

Em solos compactados, a RP pode atingir valores restritivos ao crescimento das raízes, com reflexos na produtividade das culturas (Lapen et al., 2001; Radford et al., 2001). Pesquisas têm estabelecido um possível envolvimento da RP com mensagens hormonais, que culminam com a redução na atividade estomática efotossintética das plantas em solos compactados (Davies \& Zhang, 1991). Valores de RP maiores que $2 \mathrm{MPa}$ têm sido considerados fortemente restritivos ao crescimento das raízes e das plantas (Tardieu, 1994). Embora os valores críticos de RP dependam das espécies de plantas (Materechera et al., 1991), estes podem ser distintos entre os sistemas de manejo do solo (Ehlers et al., 1983). No caso do PD, os bioporos no solo podem atuar como rotas alternativas para o crescimento das raízes numa matriz mais compactada (Ehlers et al., 1983).

Vários estudos demonstraram quea compactação superficial em PD modifica, de forma detrimental, as propriedades físicas do solo (McCoy \& Cardina, 1997; Tormena et al., 1998a; Tavares Filho et al., 2001). Em alguns solos, a RP atinge valores excessivamente elevados, e, nestes casos, alguns estudos demonstram a eficiência do revolvimento mecânico para controlar a compactação do solo (Busscher et al., 2000, 2002). Os resultados obtidos por Tormena et al. (2002) demonstram a redução da RP e o aumento da porosidade por meio da escarificação superficial do solo. Alguns inconvenientes podem advir do uso desta prática, como a redução nos teores de matéria orgânica (Oliveira et al., 2001), a qual reflete na redução da estabilidade da estrutura eem recompactação do sol o (Busscher et al., 2002).

A matéria orgânica desempenha papel importante na qual idade do solo para o crescimento das plantas. Os sistemas de manejo do solo e dos resíduos culturais influenciam os teores de matéria orgânica (Dalal \& Chan, 2001; Freixo et al., 2002). Nos sistemas com mínimo revol vimento do solo, verificase aumento nos teores de matéria orgânica do solo (Reeves, 1997), com a concentração desta na superfície (Franzluebbers, 2002a,b). Neste sentido, a estratificação da matéria orgânica no perfil é um parâmetro indicativo da qualidade do solo (Franzluebbers, 2002a) e não há relatos da sua utilização em solos brasileiros sob diferentes sistemas de manejo.

Neste contexto, o objetivo deste trabal ho foi quantificar, num Latossolo Vermel ho eutroférrico, algumas propriedades físicas, os teores e a taxa de estratificação do COS no solo, dez anos após a instalação de dois sistemas de manejo do solo.

\section{MATERIAL E MÉTODOS}

Este estudo foi realizado no campo experimental da Cooperativa Agrícola Mista Vale do Piquiri COOPERVALE, Iocalizada nomunicípio de Pal otina, estado do Paraná (Longitude $24^{\circ} 20^{\prime}$ S e Latitude $53^{\circ} 50^{\prime}$ W). O solo foi classificado como Latossolo Vermel ho eutroférrico (E mbrapa, 1999) e o rel evo é praticamente plano ou suave ondulado, com altitudes variando de 290 a $400 \mathrm{~m}$. O clima na região é do tipo $\mathrm{Cfa}$, com verões quentes e geadas pouco freqüentes, com concentração das chuvas nos meses de verão e sem estação seca definida (Godoy et al., 1976). A temperatura média anual é de $20-22^{\circ} C$, sendo a temperatura média dos meses mais quentes superior a $22^{\circ} \mathrm{C}$ e a dos meses mais frios inferior a $18^{\circ} \mathrm{C}$.

O experimento foi estabelecido em 1993 e realizado até 2002 , utilizando os seguintes sistemas de manejo de solo: (a) Plantio direto alternado com escarificação num sistema de sucessão soja-milho safrinha ou trigo (PDR), efetuando a semeadura de soja precoce no solo escarificado e semeadura de milho de segunda safra (safrinha) ou trigo sobre os restos culturais da cultura da soja; (b) Plantio direto (PD) continuamente num sistema de rotação milhotrigo-soja-aveia-soja-nabo forrageiro. Em ambos os sistemas, a semeadura foi realizada com semeadoraadubadora específica para PD. A escarificação foi realizada com escarificador de 5 hastes, espaçadas de $0,25 \mathrm{~m}$ e com profundidade efetiva de trabalho de $0,30 \mathrm{~m}$, efetuada quando o solo apresentasse consistência friável.

As determinações de COS e das propriedades físicas do solo foram feitas em duas parcelas, representativas de cada tratamento (PD ePDR) com dimensões de $60 \times 12$ m em maio/junho de 2002. No interior das parcelas, foi dimensionada uma malha quadrada constituída de 60 pontos de amostragem regionalizados. Para determinar a densidade e porosidade do solo, foram retiradas amostras com estrutura indeformada, no centro da camada de 00,15 m, utilizando amostrador e anéis com volume de $100 \mathrm{~cm}^{3}$. Após a coleta, as amostras foram acondicionadas em sacos plásticos e mantidas sob temperatura de $\pm 4{ }^{\circ} \mathrm{C}$ até serem processadas. Para determinar a porosidade total do solo, as amostras foram saturadas por $48 \mathrm{~h}$ em bandeja com água até dois terços da altura do anel. Em seguida, as amostras foram drenadas na tensão equivalente a $0,6 \mathrm{~m}$, utilizando uma mesa de tensão. Os valores de macro, micro e porosidade total do solo foram obtidos de acordo com E mbrapa (1997). Após a secagem das amostras, a densidade do solo foi determinada de acordo com Blake \& Hartge (1986).

A curva de retenção de água no solo foi feita conforme Klute (1986), adotando-se os procedimentos de Silva et al. (1994). Os valores de potencial mátrico (em módulo) e umidade foram 
ajustados por meio da equação proposta por van Genuchten (1980), utilizando o procedimento para regressão não-linear PROC NLIN (SAS, 1999).

A RP foi medida conforme Tormena \& Roloff (1996), obtendo-se valores de RP a cada 0,05 m até 0,40 m deprofundidade. Utilizou-seum penetrômetro modelo Solotest S-310, com anel dinamométrico e haste com cone metálico com área de base de $1 \mathrm{~cm}^{2}$. As amostragens foram feitas em diferentes condições de umidade do solo. Em cada tratamento e profundidade foram obtidas 180 medidas de RP, tendo sido feitas em cada ponto de amostragem três leituras de RP.

A determinação do teor COS foi efetuada em amostras deformadas obtidas em quatro camadas: $0-0,05 ; 0,05-0,10 ; 0,10-0,20$ e 0,20-0,40 m. A coleta para as três primeiras profundidades foi feita com a utilização de uma pá de corte, fracionando-se as amostras nas respectivas profundidades. A coleta na profundidade de $0,20-0,40 \mathrm{~m}$ foi feita com a utilização de um trado. As amostras de sol o foram colocadas em bandejas para secagem em estufa a $40{ }^{\circ} \mathrm{C}$, com circulação forçada por $24 \mathrm{~h}$. Após a secagem, as amostras foram passadas em peneiras com mal ha de diâmetro de $2 \mathrm{~mm}$ e determinado o COS pel o método de Walkley \& Black, adaptado por Raij et al. (1987). A taxa de estratificação foi calculada, conforme F ranzluebbers (2002a): em cada ponto amostrado, o valor de COS da camada superficial do solo $(0-0,05 \mathrm{~m})$ foi dividido pelo val or da camada subsuperficial $(0,10-0,20 \mathrm{~m})$.

Para as variáveis densidade do solo, porosidade do solo e taxa de estratificação de COS, a análise dos dados foi feita, conforme Hatcher \& Stepanski (1997), utilizando o teste t para amostras independentes na comparação dos tratamentos. Os valores absolutos do teste $t$ foram utilizados como indicadores da magnitude dos efeitos dos tratamentos nas variáveis analisadas. Os dados de RP foram analisados utilizando um val or médio em cada profundidade por época de coleta. O erropadrão da média foi utilizado para proceder à comparação dos tratamentos em cada profundidade amostrada. Adotou-se o critério de Gravetter \& Wallnau (1995) para diferenciar estatisticamente os tratamentos. As análises estatísticas foram feitas por meio do programa estatístico SAS (SAS, 1999).

\section{RESULTADOS E DISCUSSÃO}

A macroporosidade foi a variável mais influenciada pelos tratamentos, o que pode ser constatado pel o maior val or absol uto det (Quadro 1).

A Ds foi estatisticamente diferente entre os tratamentos, com os maiores valores no PD em comparação ao PDR (Quadro 1). Maiores valores de Ds e menores de macroporosidade em solos sob plantio direto também foram constatados por diversos autores (Roth et al., 1988; Hill, 1990; K lein \& Libardi, 2002). Estes resultados devem-seà menor perturbação mecânica do sol o no PD associada com tráfego de máquinas, implicando a coalescência dos agregados e, portanto, uma matriz mais densa. De acordo com os resultados obtidos por Derpsch et al . (1991), o maior valor de Ds no PD, comparado com o PDR, provavelmente, não envolve problemas de impedância mecânica ao crescimento radicular das culturas. Os autores evidenciam que, num solo similar ao deste estudo, val ores de Ds $>1,25 \mathrm{Mg} \mathrm{m}^{-3}$ é que poderiam determinar restrições mecânicas ao crescimento radicular. Neste estudo, cerca de $20 \%$ dos valores de Ds no PD e $2 \%$ no PDR foram superiores ao valor crítico relatado por Derpsch et al. (1991).

No PDR, constataram-se diferenças na porosidade do solo em comparação com o PD (Quadro 1), indicando que a utilização de escarificador no tratamento PDR proporcionou os maiores valores de macroporosidade e porosidade total $(p<0,001)$. Em relação à microporosidade, os sistemas não diferiram entre si $(p>0,05)$. Estes resultados indicam que o aumento da Ds no PD ocorreu às expensas dos poros de maior diâmetro, enquanto o aumento da porosidade e a redução da Ds no PDR decorreram do efeito da mobilização mecânica do solo com o escarificador. No PDR, os maiores valores de macroporosidade devem-se à persistência dos efeitos da mobilização do solo, que resultaram em fraturamento dos agregados e desenvolvimento de poros, notadamente os macroporos. NoPD, os valores de macroporosidade podem estar associados aos efeitos benéficos da rotação de culturas, em especial da cultura da aveia, como atestam Tormena et al. (1998a). Valores de macroporosidade suficiente para a aeração do solo na camada estudada após 10 anos de plantio direto é corroborada por Voorhees \& Lindstrom (1984), os quais afirmam serem estas condições estabel eci das após 3-4 anos de PD.

Quadro 1. Valores médios de densidade do solo (Ds), macroporosidade (Mac), microporosidade (Mic) e porosidade total (Pt) nos sistemas de manejo PD e PDR e os respectivos valores de t e $p<t$

\begin{tabular}{cclll}
\hline Tratamento & Ds & Mac & Mic & Pt \\
\hline & $\mathrm{Mg} \mathrm{m}^{-3}$ & $\mathrm{~m}^{3} \mathrm{~m}^{-3}$ & $\mathrm{~m}^{3} \mathrm{~m}^{-3}$ & $\mathrm{~m}^{3} \mathrm{~m}^{-3}$ \\
PD & 1,19 & 0,11 & 0,35 & 0,46 \\
PDR & 1,16 & 0,20 & 0,34 & 0,54 \\
$|\mathrm{t}|$ & 2,64 & 6,48 & 0,15 & 6,36 \\
$\mathrm{p}<\mathrm{t}$ & 0,0095 & 0,0001 & 0,8797 & 0,0001 \\
\hline
\end{tabular}


Apesar de os val ores médios de macroporosidade serem diferentes, o valor médio de $11 \%$ no PD foi maior que o valor crítico de $10 \%$ de poros com ar estabel ecido na literatura (Erickson, 1982; Xu et al., 1992), revelando que, mesmo sob elevados potenciais da água no solo, a difusão de $\mathrm{O}_{2}$ às raízes não é impeditiva. Num estudo em solo similar, Klein \& Libardi (2002) concluíram que, com elevados valores de macroporos, é provável que não ocorram restrições de aeração durante a redistribuição de água após chuva ou irrigação. Também Tormena et al. (1998b) verificaram que, em Latossol os argilosos com estrutura microgranular, são reduzidas as possibilidades de ser a aeração impeditiva ao crescimento das plantas. No PDR, o valor da macroporosidade foi muito el evado $\left(0,20 \mathrm{~m}^{3} \mathrm{~m}^{-3}\right)$, o que implica excessiva drenagem de água no solo.

A curva de retenção deágua do solo nos sistemas de manejo PD e PDR é mostrada na figura 1 . O modelo de van Genuchten (1980) ajustou-se adequadamente aos dados de $\theta(|\Psi|)$, demonstrado pelos elevados valores de coeficiente de deter minação $\left(R^{2}>0,98\right)$, tanto em $P D R$ quanto em $P D$. Os valores do parâmetro alfa (PD $=0,059 \mathrm{~cm}^{-1}$ e PDR $=0,102 \mathrm{~cm}^{-1}$ ) do modelo de Genuchten permitem fazer uma estimativa do potencial de entrada dear no sol o eindicam que no PD os valores deste potencial são menores do que no PDR. No $P D$, a menor retenção de água em elevados potenciais e a maior retenção no potencial de -1,5 MPa estão relacionados com a maior Ds, de acordo com os resultados obtidos por Tormena et al. (1999). Além disso, os maiores teores de CO do solo noPD resultam em maior superfície para a adsorção de água, justificando a maior retenção de água no potencial de-1,5 $\mathrm{MPa}$.

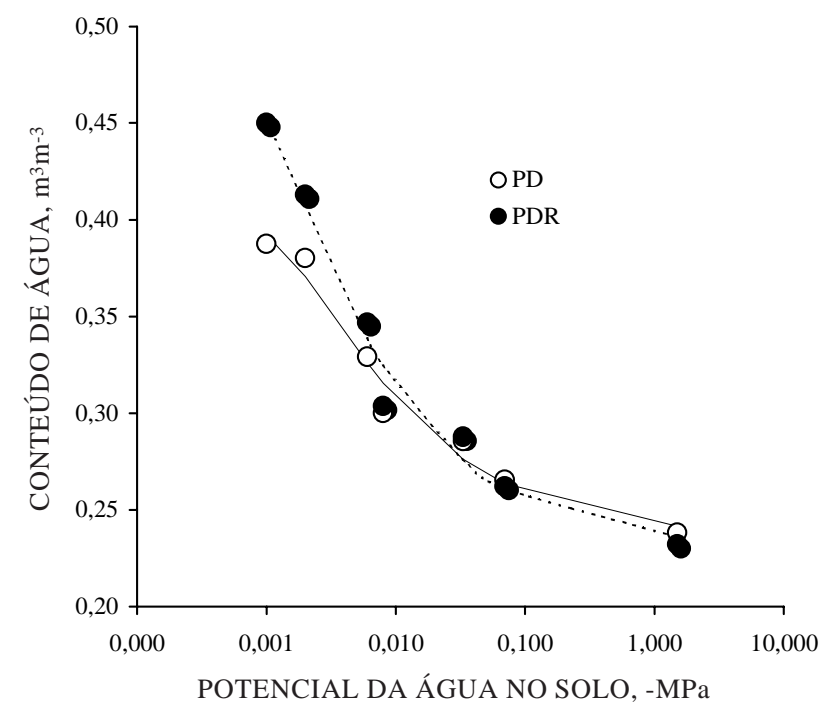

Figura 1. Curva de retenção de água no solo para os sistemas de manejo PD e PDR. As linhas referem-se ao modelo de van Genuchten (1980) ajustado aos dados.
O PD promoveu alterações na fração de poros relacionada com a retenção deágua, enquanto oPDR aumentou o volume de poros relacionados com a drenagem, de modo quea água presente nestes poros está sujeita ao rápido movimento no solo. A maior retenção deágua no PDR em potenciais maiores que -0,008 MPa demonstra a distribuição de poros de maior diâmetro, como constatado pela maior macroporosidade do solo (Quadro 1), associada ao efeito de retenção de água pelos resíduos incorporados, segundo os resultados de Quemada \& Cabrera (2002).

De acordo com as indicações de Scott $\&$ Wood (1989) e Hamblin (1982), a maior retenção de água no PDR, sob el evados potenciais, pode ser temporariamente transiente, em razão da rápida perda de água no perfil, influenciando muito pouco a disponibilidade de água às plantas, como constatado por Tormena et al. (1998b) e Klein \& Libardi (2002). Para potenciais menores que $-0,008 \mathrm{MPa}$, as curvas de retenção apresentaram poucas diferenças, sendo superiores no PD. Estes resultados estão de acordo com os de Tollner et al. (1984), que constataram ter o revolvimento do solo proporcionado aumento no número de poros drenáveis. Os valores de água disponível entre os potenciais de -0,006 MPa (capacidade de campo) e -1,5 MPa (ponto de murcha permanente), estimados a partir da função de Genuchten, foram maiores no PDR em comparação ao PD (PD $=0,0845 \mathrm{~m}^{3} \mathrm{~m}^{-3} \mathrm{e}$ $P D R=0,1030 \mathrm{~m}^{3} \mathrm{~m}^{-3}$ ). Estes resultados estão relacionados com a distribuição dos poros no PD e no PDR, mediante as maiores quantidades de poros que retêm água sob el evados potenciais no PDR ea maior retenção deágua no potencial de-1,5 MPa no PD.

$\mathrm{Na}$ amostragem realizada em $28 / 05$, com o solo mais seco, é que se constatou a maior diferença de umidade entre os tratamentos, com mai ores valores no PD em todas as profundidades avaliadas (Figura 2). J á na amostragem realizada em 07/06, os tratamentos se diferenciaram em termos dos valores de umidade nas camadas abaixo de 0,20 m, sendo o PD superior ao PDR.

Mesmo com o secamento do solo, os valores de RP no PDR mantiveram-se próximos do valor de 2,0 MPa (Figura 3), o qual éconsiderado crítico para o crescimento das raízes das plantas (Taylor et al., 1966). Estes resultados estão de acordo com os obtidos por Nevens \& Reheul (2003), em que a escarificação do solo, além de reduzir a RP, estabel eceu o controle da evolução dos seus val ores de acordo com o secamento do solo. I sto se deve à maior porosidade do solo no PDR, da qual resulta um menor número de pontos de contato entre agregados, facilitando o deslocamento e o rearranjamento destes por ocasião da medida da resistência. Por outrolado, a maior Ds na superfície do solo sob PD resulta invariavel mente em ganhos 


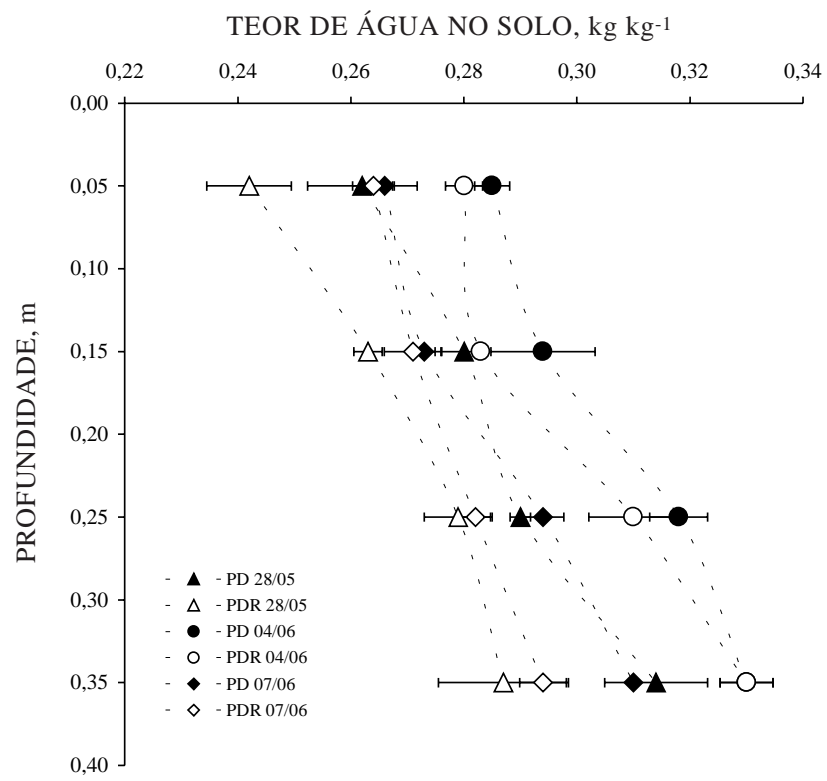

Figura 2. Umidade gravimétrica média do solo nos sistemas de manejo PD e PDR em amostragens realizadas em 28/05/2002, 04/06/2002 e 07/06/ 2002. As barras referem-se ao erro-padrão da média.

de RP com a redução da umidade do solo graças à maior coesão entre as partículas sólidas. Em solos mais compactados, resultante do mai or atrito entre as partículas, há necessidade de maior umidade no solo para facilitar o deslocamento das partículas (Silva et al., 2002). Neste sentido, a cobertura do sol o é fundamental no PD para que haja uma menor taxa de perda de água do solo (Bragagnolo \& Mielcnizuck, 1990), de modo que os valores de RP sejam mantidos em níveis não-restritivos ao crescimento das plantas. O aumento da RP com a perda deágua também foi constatado por Weaich et al. (1992).

Deforma geral, os resultados deste estudo estão em acordo com os obtidos por Tavares Filho (2001), que constataram que até $0,15 \mathrm{~m}$ o PD apresentou maiores valores de RP em relação ao preparo convencional e que de $0,15 \mathrm{~m}$ até $0,40 \mathrm{~m}$ não ocorreram diferenças estatísticas entre os tratamentos. Estes resultados confirmam a afirmativa de Derpsch et al. (1986) de que no PD a compactação do sol o émais superficial, concentrada na camada de $0-0,20 \mathrm{~m}$, contrastando com os resultados de Klein \& Libardi (2002), que verificaram aumento da compactação até $0,40 \mathrm{~m}$ de profundidade num Latossolo Vermel ho ácrico com classetextural similar.

Constatou-se que, no PDR, ocorreu aumento na quantidade média de COS em profundidade comparado com o PD (Figura 4), corroborando os resultados obtidos por I smail et al. (1994). A maior concentração de C em profundidade no PDR pode estar relacionada com a incorporação parcial dos resíduos por ocasião da escarificação do solo. Estes resultados são similares aos obtidos por Franzluebbers (2002a) e por F reixo et al. (2002), no Brasil. De acordo com F ranzl uebber et al. (1996), a não-incorporação dos resíduos, associada à menor umidade na interface solo-atmosfera, torna mais efetivo o acúmulo de COS na camada superficial do sol o no PD comparado ao PDR.

Constatou-se que a taxa média de estratificação de COS foi maior no PD comparado com o PDR (Figura 5), em consonância com os resultados de Franzl uebbers (2002a) e de Alvarez et al. (1998), o que se deve ao menor revolvimento do solo, à deposição dos resíduos culturais na superfície do solo, bem como ao efeito da rotação de culturas. Observa-se, também, que os valores máximos no PD são quase duas vezes aqueles no PDR, enquanto o contrário ocorre com os valores mínimos. Isto envolve maior variabilidadena taxa de estratificação no PD, constatada pelo coeficiente de variação de 37 \% no PD ede $19 \%$ no PDR. A avaliação dos dados indicou que $34 \%$ destes apresentaram taxas de estratificação maiores que 2, demonstrando que o CO no PD concentra-se na camada superficial do solo de 0-0,05 m.

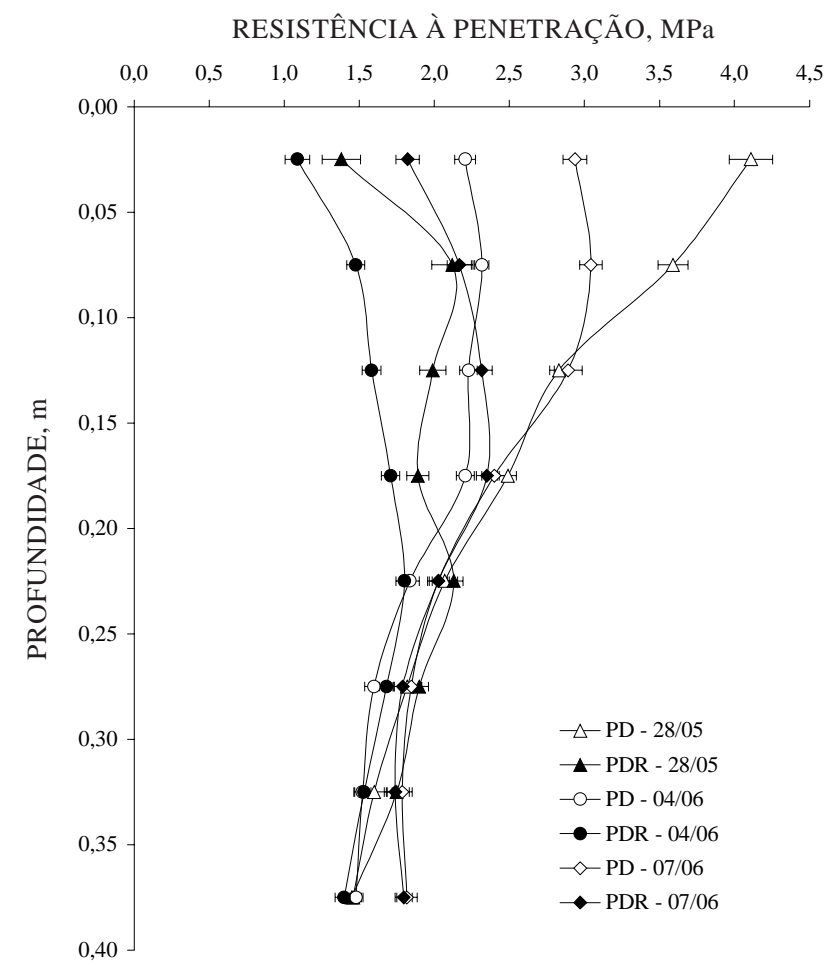

Figura 3. Valores médios de resistência do solo à penetração no PD e PDR em amostragens realizadas em 28/05/2002, 04/06/2002 e 07/06/ 2002. As barras referem-se ao erro-padrão da média e a não-sobreposição delas indica diferença significativa entre os tratamentos. 


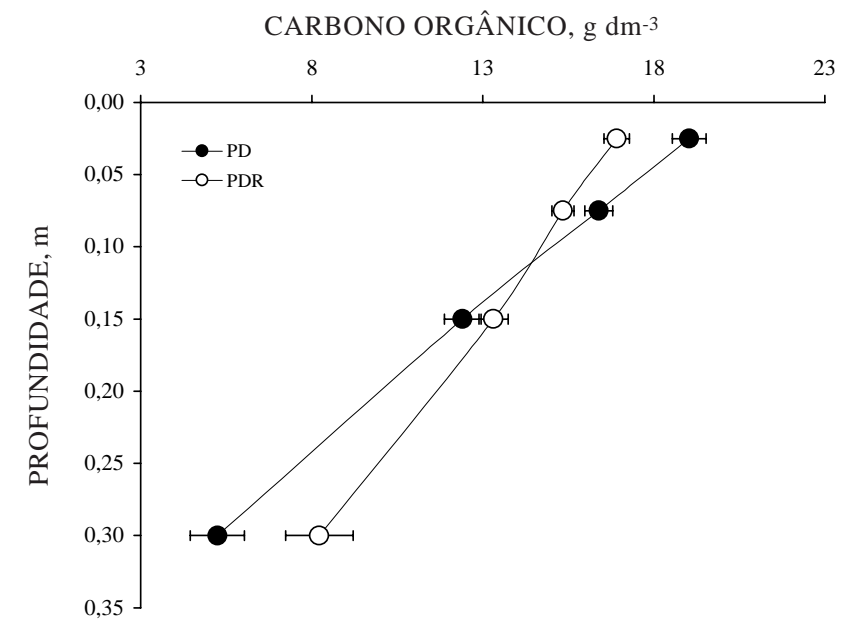

Figura 4. Distribuição dos valores médios do carbono orgânico do solo em profundidade no PD e no PDR. As barras referem-se ao erro-padrão da média e a não-sobreposição delas indica diferenças significativas entre os tratamentos.
Avaliações de produtividade da cultura da soja na safra 2002/03 indicaram maior produtividade no PD, ainda que osolo apresentasse maior Dse RP na camada de 0-0,20 m. A presença das culturas de aveia preta e nabo forrageiro no PD podeinfluenciar positivamente a qualidade física do solo, pelo fato de as raízes crescerem em profundidade e em camadas de maior compactação do solo, como revelam os resultados obtidos por Tormena $\&$ Rol off (1996) e por Muller et al. (2001). Neste sentido, a bioporosidade do sol o sob PD, anal isada com rotação de culturas planejada, resultante da formação de canais contínuos ao longo do perfil, possibilita o crescimento radicular numa matriz mais densa. Os resultados obtidos por Ehlers et al. (1983) indicam que os bioporos possi bilitam o crescimento das raízes em solos mais compactados. Além disso, estes bioporos podem criar condições de acesso às raízes da água armazenada nas camadas subsuperficiais do PD (Figura 2), indicado pel os perfis de umidade obtidos por ocasião da medida da RP.

\section{CONCLUSÕES}

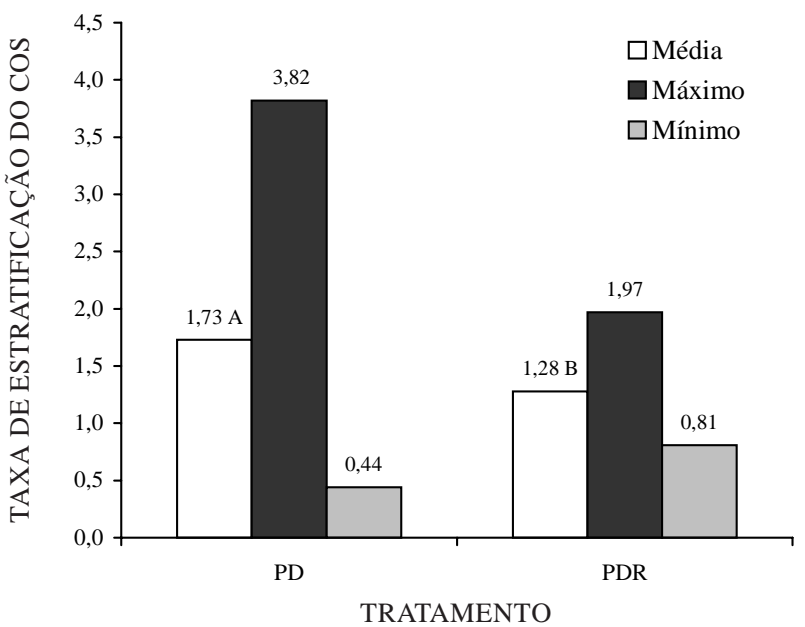

Figura 5. Valores máximos, médios e mínimos da taxa de estratificação de carbono orgânico do solo sob PD e PDR. Valores médios seguidos de letras diferentes indicam diferenças estatisticamente significativas pelo teste $t$ $(p<0,05)$.

Esta concentração de COS é importante para a qualidade do solo no PD, uma vez que diferentes tipos de estresses relacionados com aplicação de agroquímicos, fertilizantes, chuva e tráfego de máquinas incidem diretamentena superfície do sol o. Deacordo com F ranzluebber (2002b), a concentração de COS nas camadas superficiais no PD permite maior estabilidade do sistema poroso, o que pode amenizar os efeitos da compactação do solo, constatados pela mai or RP resultante da matriz mais densa do solo no PD.
1. A escarificação do solo promoveu redução na Ds e RP e el evação da macroporosidade, refletindo maiores retenções de água sob el evados potenciais. Os valores de macroporosidade foram maiores que $10 \%$ em ambos os sistemas de manejo.

2. O sistema plantio direto sem revolvimento do sol o e com rotação de culturas resultou em acúmulo de $C$ nas camadas superficiais do solo.

3. A taxa de estratificação do CO do sol o foi cerca de duas vezes maior no PD do que no PDR.

4. Com rotação de culturas, o PD apresentou a capacidade de suportar maiores restrições físicas, associado à maior taxa de acúmulo de $C$ na camada superficial do sol o em relação ao PDR. Nas condições em que o estudo foi realizado, $\mathrm{RP} \geq 2,0 \mathrm{MPa}$ não foi restritiva à cultura da soja.

\section{LITERATURA CITADA}

ALVAREZ, R.; RUSSO, M.E.; PRYSTUPA, P.; SCHEINER, J .D. $\&$ BLOTTA, L. Soil carbon pools under conventional and no-tillage systems in the Argentine Rolling Pampa. Agron. J ., 90:138-143, 1998.

BLAKE, G.R. \& HARTGE, K.H. Bulk density. In: KLUTE, A., ed. Methods of soil analysis: physical and mineralogical methods. Madison, America Society of Agronomy, 1986. p.363-375.

BOIZARD, H.; RICHARD, G.; ROGER-ESTRADE, J .; DURR, C. \& BOIFFIN, J . Cumulative effects of cropping system on the structure of the tilled layer in Northern France. Soil Till. Res., 64:149-164, 2002. 
BOONE, F.R.; van der WERF, H.M.G.; KROESBERGEN, B.; HAAG, B.A. TEM \& BOERS, A. The effect of compaction of arable layer in a sandy soils on the growth of maize for silage. I. Critical potentials in relation to soil aeration and mechanical impedance. Neth. J. Agric. Res., 34:155-171, 1986.

BRAGAGNOLO, N. \& MIELNICZUK, J . Cobertura do solo por palha de trigo e seu relacionamento com a temperatura e umidade do solo. R. Bras. Ci. Solo, 14:369-374, 1990.

BUSSCHER, W.J .; BAUER, P.J . \& FREDERICK, J.R. Recompaction of a coastal loamy sand after deep tillage as a function of subsequent cumulative rainfall. Soil Till. Res., 68:49-57, 2002.

BUSSCHER, W.J .; FREDERICK, J .R. \& BAUER, P.J . Timing effects of deep tillage on penetration resistance and wheat and soybean yield. Soil Sci. Soc. Am. J ., 64:999-1003, 2000.

CASSEL, D.K. \& NIELSEN, D.R. Field capacity and available water capacity. In. KLUTE, A., ed. Methods of soil analysis: physical and mineralogical methods. 2.ed. Madison, America Society of Agronomy, 1986. p.901-926.

DALAL, R.C. \& CHAN, K.Y. Soil organic matter in rainfed cropping systems of the Australian cereal belt. Austr. J . Soil Res., 39:435-464, 2001.

DAVIES, W.J . \& ZHANG, J . Root signals and the regulation of growth and development of plants in drying soil. Ann. Rev. Plant Phys. Plant Mol. Biol., 42:55-76, 1991.

DE MARIA, I.C.; CASTRO, O.M. \& SOUZA DIAS, H. Atributos físicos do solo e crescimento radicular de soja em Latossolo Roxo sob diferentes métodos de preparo do solo. R. Bras. Ci. Solo, 23:703-709, 1999.

DEFOSSEZ, P. \& RICHARD, G. Models of soil compaction due totraffic and their evaluation. Soil Till. Res., 67:41-64, 2002.

DERPSCH, R.; ROTH, C.H.; SIDIRAS, N. \& KÖPKE, U. Controle da erosãono Paraná, Brasil: Sistemas de cobertura do solo, plantio direto e preparo conservacionista do solo. Londrina, Fundação I nstituto Agronômico do Paraná, 272p. 1991.

DERPSCH, R.; SIDIRAS, N. \& ROTH, C.H. Results of studies from 1977 to 1984 to control erosion by cover crops and notillage techniques in Parana, Brazil. Soil Till. Res., 8:253263, 1986.

EHLERS, W.W.; KOPKE, F.; HESSE, F. \& BOHM, W. Penetration resistance and growth root of oats in tilled and untilled loess soil. Soil Till. Res., 3:261-275, 1983.

EMPRESA BRASILEIRA DE PESQUISA AGROPECUÁRIA EMBRAPA. Centro Nacional de Pesquisa de Solos. Manual de métodos de análise de solo. 2 ed. Rio de J aneiro, 1997. $212 p$.

EMPRESA BRASILEIRA DE PESQUISA AGROPECUÁRIA EMBRAPA. Sistema brasileiro de classificação de solos. Rio de J aneiro, 1999. 412 p.

ERICKSON, A.E. Tillage effects on soil aeration. In: PREDICTING TILLAGE EFFECTS ON SOIL PHYSICAL PROPERTIES AND PROCESESS, 1982. Madison. Proceedings. America Society of Agronomy, 1982. p.91-104.
FRANZLUEBBERS, A.J . Soil organic matter stratification ratio as an indicator of soil quality. Soil Till. Res., 66:95-106, 2002a.

FRANZLUEBBERS, A.J. Water infiltration and soil structure related to organic matter and its stratification with depth. Soil Till. Res., 66:197-205, 2002b.

FRANZLUEBBERS, A.J .; ARSHAD, M.A. \& RIPMEESTER, J .A. Alterations in canola residue composition during decomposition. Soil Biol. Biochem., 28:1289-1295, 1996.

FREIXO, A.A.; MACHADO, P.L.O.A.; GUIMARÃES, C.M.; SILVA, C.A. \& FADIGAS, F.S. Estoques de carbono e nitrogênio e distribuição das frações orgânicas de um Latossolo do cerrado sob diferentes sistemas de cultivo. R. Bras. Ci. Solo, 26:425-434, 2002.

GODOY, H.; CORREA, A.R. \& SANTOS, D. Clima do Paraná. In: Fundação Instituto Agronômico do Paraná. Manual Agropecuário para o Paraná. 1976.

GRAVETTER, F.J \& \& WALLNAU, L.B. Statistics for the behavioral sciences. Londrina, 2.ed. St. Paul, West Publishing, 1995. 429p.

HAMBLIN, A.P. Soil water behaviour in response to changes in soil structure. J . Soil Sci., 33:375-386, 1982.

HATCHER, L. \& STEPANSKI, E.J . A step-by-step approach to using the SAS System for Univariate and Multivariate Statistics, Cary, SAS Institute, 1997. 552p.

HILL, R.L. Long-term conventional and no-tillage effects on selected soil physical properties. Soil Sci. Soc. Am. J., 54:161-166, 1990.

ISMAIL, I.; BLEVINS, R.L. \& FRYE, W.W. Long-term no-tillage effects on soil properties and continuous corn yields. Soil Sci. Soc. Am. J ., 58:193-198, 1994.

KLEIN, V.A. \& LIBARDI, P.L. Densidade e distribuição do diâmetro dos poros de um Latossolo Vermelho sob diferentes sistemas de uso e manejo. R. Bras. Ci. Solo, 26:857-867, 2002

KLUTE, A. Water retention: laboratory methods. In: KLUTE, A., ed. Methods of soil analysis - physical and mineralogical methods. Madison, America Society of Agronomy, 1986. p. 635-660.

LAPPEN, D.R.; TOPP, G.C.; GREGORICH, E.G.; HAYHOE, H.N. \& CURNOE, W.E. Divisive field-scale associations between corn yields, management, and soil information. Soil Till. Res., 58:193-206, 2001.

MATERECHERA, S.A.; DEXTER, A.R. \& ALSON, A.M. Penetration of very strong soils by seedling of different plant species. Plant Soil, 135:31-41, 1991.

MCCOY, E.L. \& CARDINA, J. Characterizing the structure of undisturbed soils. Soil Sci. Soc. Am. J ., 61:280-286, 1997.

MULLER, M.M.L.; CECCON, G. \& ROSOLEM, C.A. Influência da compactação do solo em subsuperfície sobre o crescimento aéreo e radicular de plantas de adubação verde de inverno. R. Bras. Ci. Solo, 25:531-538, 2001. 
NEVENS, F . \& REHEUL, D. The consequences of wheel-induced soil compaction and subsoiling for silage maize on a sandy loam soil in Belgium. Soil Till. Res., 70:175-184, 2003.

OLIVEIRA, J .O.A.P.; VIDIGAL FILHO, P.S.; TORMENA, C.A.; PEQUENO, M.G.; SCAPIM, C.A.; MUNIZ, A.S. \& SAGRILO, E. Influência de sistemas de preparo do sol o na produtividade da mandioca (Manihot esculenta, Crantz). R. Bras. Ci. Solo, 25:443-450, 2001.

QUEMADA, M. \& CABRERA, M.L. Characteristics moisture curves and maximum water content of two crop residues. Plant Soil, 238:295-299, 2002.

RADFORD, B.J .; YULE, D.F.; MCGARRY, D. \& PLAYFORD, C. Crop responses to applied soil compaction and to compaction repair treatments. Soil Till. Res.,61:157-166, 2001.

RAIJ , B. van; QUAGGIO,J .A.; CANTARELLA, H.; FERREIRA, M.E.; A.S. \& BATAGLIA, O.C. Análise química do solo para fins defertilidade. Campinas, Fundação Cargill, 1987. 170p.

REEVES, D.W. The role of soil organic matter in maintaining soil quality in continuous cropping systems. Soil Till. Res., 43:131-167, 1997.

ROTH, C.H.; MEYER, B.; FREDE, H.G. \& DERPSCH, R. Effect of mulch rates and tillagesystems on infiltrability and other soil physical properties of an Oxisol in Paraná, Brazil. Soil Till. Res., 11:81-91, 1988.

SAS INSTITUTE. Statiscal Analysis System I nstitute. Procedure guide for personal computers. Version 5, Cary, 1999.

SCOTT, H.D. \& WOOD, L.S. I mpact of crop production on the physical status of a Typic Albaqualf. Soil Sci. Soc. Am. J ., 53:1819-1825, 1989.

SILVA, A.P.; KAY, B.D. \& PERFECT, E. Characterization of the least limiting water range. Soil Sci. Soc. Am. J ., 58:17751781, 1994.

SILVA, V.R.; REINERT, D.J . \& REICHERT, J.M. Fatores controladores da compressibilidade de um Argissolo Vermelho-Amarelo distrófico arênico e de um Latossolo Vermel ho distrófico típico. II - Grau de saturação em água. R. Bras. Ci. Solo, 26:9-15, 2002.

TARDIEU, F. Growth and functioning of roots and to root systems subjected to soil compaction. Towards a system with multiple signaling. Soil Till. Res., 30:217-243, 1994.
TAVARES FILHO, J .; BARBOSA, G.M.C.; GUIMARÃES, M.F. \& FONSECA, I.C.B. Resistência do solo à penetração e desenvolvimento do sistema radicular do milho (Zea mays) sob diferentes sistemas de manejo em um Latossolo Roxo. R. Bras. Ci. Solo, 25:725-730, 2001.

TAYLOR, H.M.; ROBERSON, G.M. \& PARKER J r., J .J . Soil strength-root penetration relations to medium to coarsetextured soil materials. Soil Sci., 102:18-22, 1966.

TOLLNER, E.W.; HARGROVE, W.L. \& LANGDALE, G.W. Influence of conventional and no-tillage practices on soil physical properties in the southern Piedmont. J . Soil Water Conserv., 39:73-76, 1984

TORMENA, C.A. \& ROLOFF, G. Dinâmica da resistência à penetração de um solo sob plantio direto. R. Bras. Ci. Solo, 20:333-339, 1996

TORMENA, C.A.; BARBOSA, M.C.; COSTA, A.C.S. \& GONÇALVES, A.C.A. Densidade, porosidade eresistência à penetração em Latossolo Vermelho distrófico cultivado sob diferentes sistemas de preparo do solo. Sci. Agríc., 59:795-801, 2002.

TORMENA, C.A.; ROLOFF, G. \& SÁ, J.C.M. Propriedades físicas do solo sob plantio direto influenciado por calagem, preparo inicial e tráfego. R. Bras. Ci. Solo, 22:301-309, 1998a.

TORMENA, C.A.; SILVA, A.P. \& LIBARDI, P.L. Caracterização do intervalo hídrico ótimo de um Latossolo Roxo sob plantio direto. R. Bras. Ci. Solo, 22:573-581, 1998b.

TORMENA, C.A.; SILVA, A.P. \& LIBARDI, P.L. Soil physical quality of a Brazilian Oxisol under twotillagesystems using the least limiting water range approach. Soil Till. Res., 52:223-232, 1999.

van GENUCHTEN, M. Th. A closed form equation for predicting hydraulic conductivity of unsaturated soils. Soil Sci. Soc. Am. J ., 44:892-898, 1980.

VOORHEES, W.B. \& LINDSTROM, M.J . Long-term effects of tillage method on soil tilth independent of wheel traffic compaction. Soil Sci. Soc. Am. J ., 48:152-156, 1984.

WEAICH, K.; BRISTOW, K.L. \& CASS, A. Preemergent shoot growth of maize under different drying conditions. Soil Sci. Soc. Am. J ., 56:1272-1278, 1992.

XU, X.; NIEBER, J.L. \& GUPTA, S.C. Compaction effects on the gas diffusion coefficients in soil. Soil Sci. Soc., Am. J., 56:1743-1750, 1992. 